\title{
Traumatic recto-vaginal fistula after consensual sexual activity: a case series.
}

Kathryn Waite, Claire Webster, Sharad Karandikar \& Doug Bowley

Heartlands Hospital, Heart of England NHS Foundation Trust, Birmingham UK

\section{Background}

Severe genital injury most often occurs with rape or assault by penetration with an object, rather than consensual activity. However, absence of genital injury does not mean that rape or serious sexual assault did not occur. We highlight the stories of two woman that demonstrate severe genital injuries can occur even after consensual sexual activity. Written consent from both patients has been obtained for publication.

\section{Story \#1}

A 45-year-old woman presented with vaginal

bleeding and sepsis; her abdomen was distended and rigid. Erect CXR indicated perforated viscus with pneumoperitoneum (Figure 1). She had noticed faecal matter per vaginam. Laparotomy revealed extensive faecal peritonitis due to a $10 \mathrm{~cm}$ tear to the antero-lateral rectal wall with a defect in the adjacent vaginal wall. The injury had occurred when, under the influence of alcohol but with mutual consent, sex toys had been simultaneously inserted (double penetration) into the vagina and anus. Laparotomy and

Hartmann's colectomy was required.

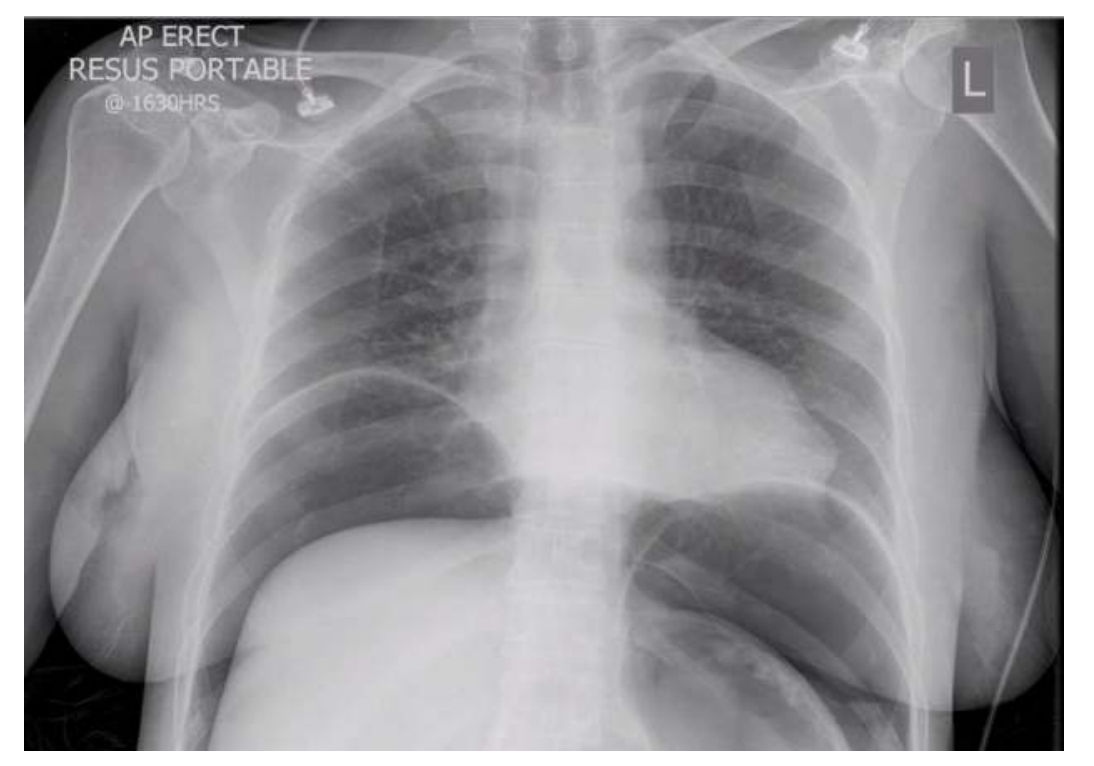

Figure 1: Resuscitation room erect CXR demonstrating free air within the abdominal cavity
Story \#2

A 19-year-old woman had experienced bleeding per vaginam after 'vanilla' intercourse. Seven days later, she noticed faeculent material per vaginam. On examination, a $2 \mathrm{~cm}$ defect in the posterior vaginal wall was identified with a corresponding rent in the low rectum.

Defunctioning stoma was raised and a successful elective repair undertaken 5 months later.

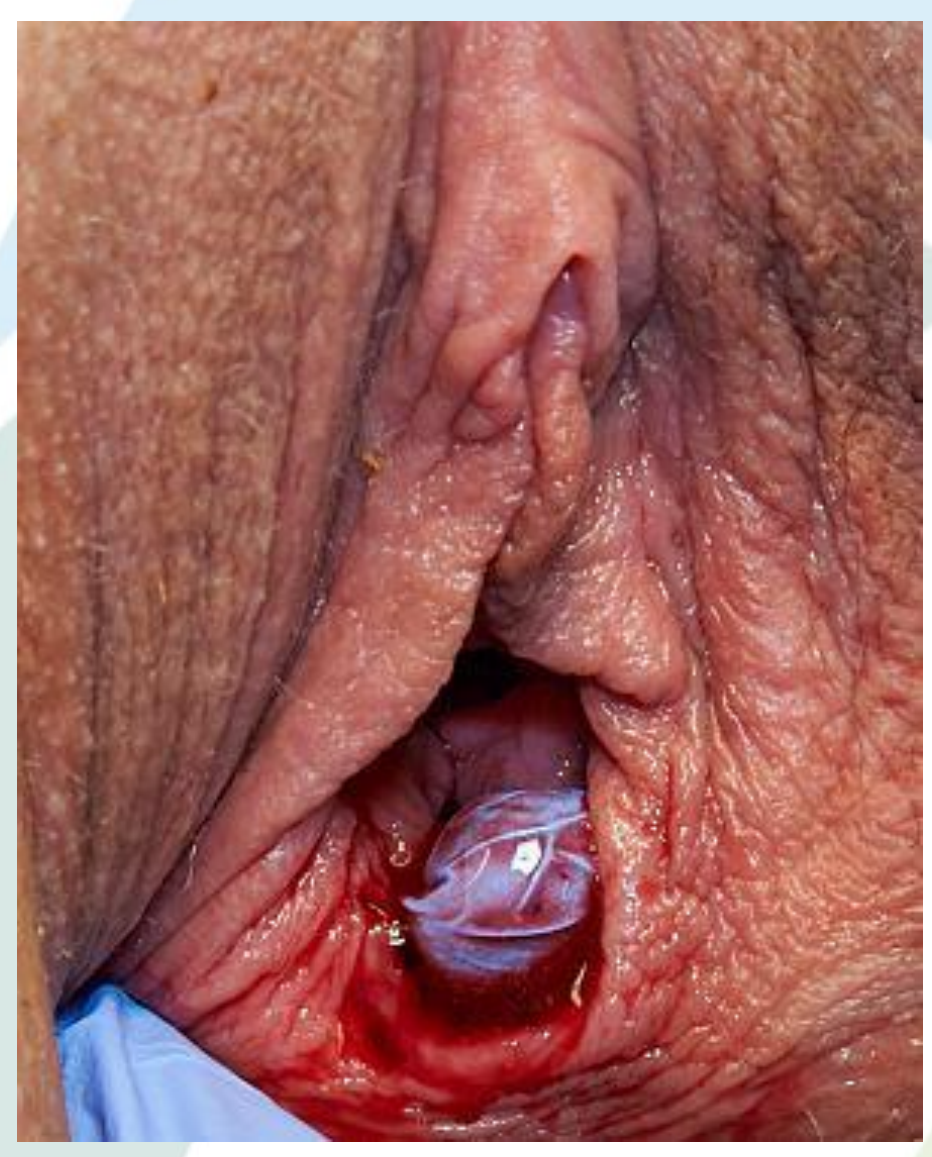

Figure 2: Clinical photo shows the tip of a gloved finger demonstrating the recto-vaginal fistula

\section{Learning Points}

Recognition that severe genital injury can occur following consensual sex is important for healthcare professionals; the learning from our patients adds to our understanding of the potentially harmful result of human sexual activity. In western society, there has been a large rise in the use of sex toys and it is

widely recognised that these adjuncts can enhance sexual functioning for both women and men. However, our first patient's story highlights the public health implications of sex toy usage. The industry is

unregulated with a distinct absence of health information on packaging and a dearth of educational resources for the public. Healthcare workers in this area should advocate for enhanced public education and increased regulation of the lucrative sex toy market. 